\title{
La posibilidad de hacer. La creación sonora en el espacio social
}

\author{
The Possibility of Doing. \\ The Sonoric Creation in the Social Space
}

María Andueza Olmedo

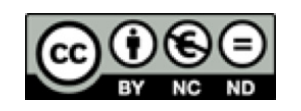

Esta obra está bajo una licencia Creative Commons Reconocimiento-No comercial-Sin Obra Derivada 


\title{
La posibilidad de hacer. La creación sonora en el espacio social
}

\author{
The Possibility of Doing. \\ The Sound Creation in the Social Space
}

\author{
María Andueza Olmedo ${ }^{1}$ \\ Universidad Internacional de la Rioja \\ España
}

Recibido: 23 de agosto del 2017 Aprobado: 17 de octubre del 2017

\begin{abstract}
Resumen
Este artículo se centra en la creación sonora, en particular en la realizada con carácter eventual en el espacio público y, más específicamente, en aquella que fija su atención en su condición contextual. Considera la facultad de estas acciones para procurar las condiciones para un conocimiento personal y compartido, atendiendo a las situaciones que provoca y a la multiplicidad de conocimientos posibles que pueden surgir de ellas. El artículo parte de la reflexión que hace Jonathan Sterne sobre los modos de diseminar el conocimiento de los estudios sonoros en The Sound Studies Reader y propone, a través del análisis de un grupo de acciones, fijarse en el estadio anterior al conocimiento ya producido con obras cuyo modus operandi identifica determinadas cuestiones contextuales y las problematiza a través del elemento sonoro y de la escucha como motor del conocimiento.

Palabras clave: creación sonora; espacio público; arte; espacialidad; escenografía de la escucha
\end{abstract}

1 Profesora adjunta.en la Universidad Internacional de la Rioja. Acreditada en la figura de Profesor Contratado Doctor por la Agencia Nacional de Evaluación de la Calidad y Acreditación (ANECA). Doctora, con mención europea, por la Universidad Complutense de Madrid. Correo electrónico: maria.andueza@unir.net

ESCENA. Revista de las artes, 2018, Vol. 78, Núm. 1 (julio-diciembre), pp. 70-82. ISSN 2215-4906 


\begin{abstract}
This essay addresses eventual sound creation in public spaces and specifically that one which focuses attention in the contextual condition. It considers the attributes of this field of sound creation that propitiate the conditions for a multiple, individual and shared knowledge. The paper starts from the problem of knowledge as presented by Jonathan Sterne in The Sound Studies Reader and considers the modus operandi of a group of pieces that work identifying the context and problematizing it through sound and listening as motors for knowledge.
\end{abstract}

Keywords: sound art; public space; art; spatiality; listening scenography 
En su introducción a la compilación de textos reunidos en The Sound Studies Reader, Jonathan Sterne (2012) plantea el conocimiento como "problema" para el campo de los estudios sonoros. Se pregunta Sterne por los procedimientos para abordar la diseminación de dicho conocimiento entendiendo que son muchos y variados y que, por lo tanto, es importante preguntarse por la convivencia de los saberes que de ellos se derivan y por la posición que ocupa el conocimiento, o los conocimientos, obtenidos a través de lo sonoro, con relación a los discursos dominantes y a aquellos minoritarios, tratando de reconocer la red que esos conocimientos tejen con ellos. El problema para Sterne pasa por comprender que los estudios sonoros, sean estos de la categoría que sean, están condicionados por el conjunto de conocimientos sobre el sonido que a su vez estos motivan, utilizan y ponen práctica. Como también implica reconocer que ese conocimiento está influenciado por las tradiciones y los métodos heredados de los propios campos de estudio sonoro y por aquellos conocimientos prácticos y/o formales que encuentra en los objetos que estudia. Es decir, Jonathan Sterne (2012) expone que el conocimiento que resulta de las prácticas sonoras no está exento de las influencias y las aportaciones venidas de otros campos y que, por lo tanto, debe ser entendido con relación a ellos y nunca de forma independiente o aislada de los mismos. En consecuencia, como él mismo indica, los estudios sonoros son una forma de pensamiento conjunto sobre el sonido y la cultura.

En la línea de lo planteado por Sterne, fijarse precisamente en la formulación misma de dicho problema -lo que implica situarse un paso más atrás del conocimiento ya producido y hacerlo en las condiciones que lo propician- emplaza a la creación sonora como un acontecimiento más de la cultura contemporánea, como un proceso que arroja luz sobre la relevancia que esta tiene en los discursos actuales. Al menos así sucede si nos situamos en la esfera de lo público, en la que la creación sonora aparece como una respuesta directa a los estímulos de lo urbano -o de lo público en términos más amplios- adquiriendo formas y metodologías sonoras que son acordes a la identificación previa de un problema, con el que dialoga a través de diferentes contextos, casos o acciones. Además de esto, por darse el diálogo en el contexto de lo público, la creación sonora busca y demanda a otros interlocutores: oyentes, audiencias, pero, sobre todo, ciudadanos, transeúntes o habitantes de lo público para los que procurará las condiciones de una experiencia. En otras palabras, no hablaremos de un conocimiento elaborado, que se va a producir -o tal vez lo haga- en un momento posterior, sino de las condiciones para el mismo.

\section{La apertura del espacio}

En lugar de pensar cómo diseminar el conocimiento a través de la creación sonora en el espacio público -o además de ello-, tiene sentido analizar la potencialidad de la creación sonora para abrir procesos en el espacio público que generan en los otros escenarios 
de análisis, estudio y crítica. Así, dejamos de pensar lo que la creación sonora hace o produce en última instancia, sino que se fija la atención en su capacidad de hacer, en su potencialidad como agente disruptivo, en su facultad de producir un cambio de registro, o de percepción, en la esfera pública a distintos niveles. Enmarcada en la posibilidad y no tanto en lo ya producido, entabla un coloquio, desde lo sonoro, con los procesos que operan en el espacio público. Y de este modo, llega a formas de conocimiento no unidireccionales, sino compuestas de una multiplicidad de aproximaciones que son a su vez propias de la configuración de la opinión pública de la que participan.

Un ejemplo temprano de todo esto lo encontramos en la intervención radiofónica que llevó a cabo, en 1973, Suzanne Lacy como parte del proyecto Three Weeks in May. El proyecto identificaba y denunciaba, a través de diversas acciones y performances que recurrían a diferentes metodologías y recursos artísticos, la violencia sexual ejercida contra mujeres en la ciudad de Los Angeles. Procuraba, mediante acciones como la creación de un mapa o manifestaciones con pancartas informativas, oportunidades para contribuir a la concienciación misma del problema. Asimismo, a través de estas acciones, el proyecto de Lacy ofrecía a las víctimas herramientas para la resolución y superación de la intimidación o las violaciones sufridas. Parte de la estrategia diseñada por la artista en este proyecto pasaba por implicar a las víctimas y a los medios en las diversas acciones, como también a figuras relevantes de la opinión pública y la política. Así, se hacía presente en el espacio público la denuncia del tema persiguiendo con ello que cada acción tuviera un impacto mayor en el propio contexto que visualizaba. Como parte de Three Weeks of May, Lacy realizó una acción en la emisora de radio KPFK de Los Angeles. La intervención tuvo lugar en el marco del programa semanal de media hora Close Radio y consistió en la lectura continuada de los atestados policiales correspondientes a las violaciones a mujeres sucedidas los días previos a la acción. La lectura de los documentos realizada como un acto consciente y decidido con voz clara y distante devolvía al lenguaje descriptivo y puramente informativo con el que fueron escritos la presencia de los cuerpos violentados. A través de la voz se corporeizaban en el mensaje impersonal forzando así una cierta identificación del oyente con el tema de la violencia sexual.

Cuando Lacy elige realizar esta acción en el medio radiofónico, posibilita que esos cuerpos a los que hacen alusión los informes se hagan presentes y aparezcan en sentido figurado en múltiples y diversos espacios: desde un hogar a una peluquería, pasando por una oficina, un taller mecánico, un taxi o un bar. El sonido de la voz de Lacy en esta acción lleva a la escucha más allá del objeto escuchado, situando al oyente, como afirma Salomé Voeghelin (2011), en la producción de lo percibido, lo que despliega en este caso concreto -en la diversidad de posibles oyentes- la complejidad del problema abordado: 
La invisibilidad del sonido de la radio permite una variedad de percepciones. El oyente se convierte en productor, inventando su propia realidad eventual entre lo que escucha y el espacio-tiempo de su percepción. Esta escucha usa la oscuridad de la radio como caverna, llena de sonido. Aquí, ninguna imagen mantiene al oyente apegado a una verdadera sensación de realidad, y por lo tanto ninguna sensación de irrealidad limita su imaginación (Voeghelin, 2011, p. 38).

Al ejecutar esta acción en la radio, Lacy está jugando precisamente con el modo en que funcionan los medios, penetrando en el espacio del otro. A partir de ahí, busca provocar una reacción, llegar al interés de los oyentes, construir la experiencia de un problema a partir de su escucha. Sin límites, como afirma Voegelin, y sin reducirlo a los datos objetivos de las víctimas y los ejecutores, sino a partir de ellos generando una conciencia en el sujeto como primer paso para una conciencia colectiva.

La obra se convierte así en un medio para la experiencia, donde el artista actúa como un activista y la obra como catalizadora de la experiencia². La misma Suzanne Lacy (1994b) explica diferentes formas de aproximase a la creación en el espacio público, adoptando como artista diferentes roles que va a identificar como experimentador, informador, analista o activista. En este caso, aprovechando la oportunidad que le dio la ventana del programa de radio, Lacy ocupa el espacio íntimo de la escucha. Penetra con su voz en el espacio del otro, que será quien construya el significado de la pieza y con ello genere un cierto grado de conocimiento sobre el problema. No resulta tan impactante en esta obra el cómo está realizada, como el qué está transmitiendo desde esa ocupación temporal del medio radiofónico. Son, como afirma la propia Lacy (1994a), "obras que no se construyen sobre una tipología de los materiales, espacios o medios artísticos sino más bien sobre conceptos de audiencia, relación, comunicación e intención política" (p.19).

No obstante, aunque la pieza no se construya sobre el hecho sonoro, puesto que Suzanne Lacy hace un uso reiterado de este medio (también lo ha usado en otras piezas como The Cristal Quilt (1987); The Whisper, the Wave, the Wind (1984); o The Roof is son Fire (1994) reviste interés detenerse en ello y observar que efectivamente tal y como afirma la artista, el valor de lo sonoro no reside en su propia condición sonora sino en lo que esta provoca al sumarse al resto de vectores que participan de la obra. Lacy toma el espacio-tiempo radiofónicos como una oportunidad para tergiversar su transcurso habitual, ocupando el tiempo de escucha con la lectura de los informes policiales. Así consigue, a través de lo sonoro, orientar el propósito de la acción hacia las tensiones que potencialmente provocará en su encuentro con lo ajeno, interfiriendo en otros espacios y tiempos diferentes a los de la artista y las protagonistas del proyecto.

2 Véase la explicación que de todo ello se hace en Blanco (2001).

ESCENA. Revista de las artes, 2018, Vol. 78, Núm. 1 (julio-diciembre), pp. 70-82. ISSN 2215-4906 


\section{La ocupación como forma de resistencia}

Si bien en esta pieza de Lacy la ocupación se da en unos términos de virtualidad espacial, en otras obras en las que la artista trabaja en el espacio público la ocupación se hace literal, tomando un espacio, apoderándose de un territorio, alterando su uso y forzando un encuentro. La ocupación se produce así como una forma de resistencia que se materializa en los cuerpos resonantes o en la irrupción de procesos sonoros que, temporalmente, invaden o interfieren otros a los que resignifican. Así sucedió por ejemplo en la intervención The Whisper, the Wind, the Wave (1984) en la que Lacy congregó a mujeres de la tercera edad en una playa de California con el objeto de conversar. La sola disposición del espacio ya suponía una irrupción en el mismo, no obstante, el motivo sonoro -la conversación- adquiría una posición protagonista en el lugar como interferencia que conseguía ordenar a su vez la relación con las audiencias a partir de ella. En primera instancia, al público solo se le permitía escuchar y mirar desde la distancia, sumado al rumor que pudieran percibir desde una cierta lejanía, se escuchaba también una remezcla de las conversaciones. En un momento posterior la audiencia podía caminar entre las mesas escuchando desde la cercanía las conversaciones y participando así mediante su contemplación activa del evento creado por Lacy.

La ocupación tenía lugar en esta intervención en distintos niveles: por un lado, había una evidente ocupación mediante la toma del espacio por la presencia del grupo de mujeres. Por otro lado, sucedía una ocupación al nivel de la escucha espacial y social al tomar posesión del espacio con sus voces habitualmente silenciadas. Asimismo, como suma de todo ello se producía una ocupación desde el extrañamiento al hacerse presente con un carácter eventual y celebratorio una acción ajena al espacio intervenido.

\section{La fiesta como liberación}

Acciones como estas de Lacy anticipan o recuerdan a su vez las ocupaciones del espacio público de movimientos como Reclaim the Streets o las Love Parade donde la ocupación de la calle, ejercida por los cuerpos, da lugar a formatos festivos en los que la música juega asimismo un papel protagonista. La fiesta, mediada por la música, es una forma de ejercer la apropiación del espacio y un modo de considerarlo como bien comunal, procurando una suerte de vivencia compartida del espacio público que lo hace aparecer como una plataforma susceptible de ser apropiado. La música, por su parte, como catalizadora de esa experiencia, adquiere un papel dominante que procura el uso del espacio público como lugar de expresión.

Si bien la ciudad no está diseñada para bailar, como tampoco lo está una playa para organizar una reunión de mujeres sentadas en mesas conversando (como en el caso de The Wispher... de Lacy) la acción de escuchar música en la calle y bailar, o de pasear entre 
grupos de personas para escuchar explícitamente sus conversaciones, carga poética y simbólicamente al espacio de un potencial que acrecienta su valor como bien público, como un espacio para ser usado y no solo transitado. Un espacio que, de este modo, responde a las necesidades expresadas por la comunidad, que no aparece diseñado exclusivamente como un bien que está disponible para todos, sino del que se dispone activamente. Como afirma el sociólogo español Manuel Delgado (2008), "la calle o la plaza, en tanto que espacios públicos, no pueden conocer sino usuarios, es decir, individuos que se apropian de ellas en tanto que las usan y sólo mientras lo hacen" (párr. 1). El espacio público, por tanto, solo puede apropiarse temporalmente lo que hace todavía más interesante la temporalidad del sonido como herramienta o como vía para la apropiación y el reconocimiento del espacio público.

Acercando estas cuestiones al ámbito artístico y sonoro, y al momento contemporáneo, merece la pena detenerse en el trabajo del colectivo Grupal Crew Collective que sitúan su práctica en los campos de la música, la fiesta y el arte público. Desde estos tres ámbitos desafían al sistema de la producción cultural, alterando el uso de los espacios que intervienen a través de la fiesta. Las acciones así concebidas y estructuradas funcionan como un gesto político que empodera a los individuos en el espacio en el que la practican. Escuchar música y bailar en el espacio público implica atender y moverse conforme a otros criterios que no son los habituales de los espacios públicos ni urbanos. De este modo, la fiesta, la música, el baile abren el espacio a otros tiempos y a otras formas de actuar. En el año 2013, por ejemplo, crearon en ZAA Soundsystem un sistema de audio portátil silencioso que trasladaron por las calles del centro de la ciudad de Madrid. Poco tiempo antes, el Ayuntamiento había decretado zonas de protección acústica especial en el centro de la ciudad, regulando la amplificación del sonido en el espacio público y sometiendo a toda actuación musical susceptible de suceder en esas zonas a una autorización municipal. Esta ordenanza trajo consigo que los artistas callejeros debieran pasar un casting mediante el que conseguían la licencia para actuar en el espacio público, eliminando con este acto la diversidad y la espontaneidad propias de estas actuaciones en las calles, plazas y parques de la ciudad.

En ZAA Soundsystem los miembros de Grupal Crew Collective pincharon música por las calles del centro de Madrid provocando escenas muy significativas: grupos pequeños de tres o cuatro individuos bailando con cascos en la calle sin que otros transeúntes pudieran escuchar la música que les empujaba a mover sus cuerpos. La fiesta tan habitual de esta zona de Madrid se trasladaba así en pequeñas dosis al espacio público esquivando la reciente regulación y procurando un encuentro con los procesos habituales de la ciudad y los ciudadanos que la transitan. Se consumaba de este modo, a través de la fiesta con el elemento sonoro como motor generatriz, el disfrute del espacio público y la libertad de expresión en él. 
Nada difiere lo que se produce en esta acción de Grupal Crew Collective de lo que el escritor y ensayista estadounidense, Hakim Bey, denominó en su ensayo de 1991 Zonas Temporalmente Autónomas (TAZ en sus siglas en inglés). Estas zonas se refieren a ocupaciones temporales del espacio público con el objeto de esquivar estructuras de control social, tal y como sucedía en Reclaim the Streets impidiendo el tráfico rodado o como sucedía también en la respuesta de Grupal Crew Collective a la ordenanza del ruido impuesta por la administración pública. La inmediatez y la eventualidad son claves para entender el valor que tienen estas manifestaciones, como forma de irrupción en lo público. Así lo explica Bey en su texto, pues indica que

El TAZ es como una revuelta que no se engancha con el Estado, una operación guerrillera que libera un área -de tierra, de tiempo, de imaginación- y entonces se autodisuelve para reconstruirse en cualquier otro lugar o tiempo, antes de que el Estado pueda aplastarla. Puesto que el Estado tiene más que ver con la Simulación que con la substancia, el TAZ puede ocupar estas áreas clandestinamente y llevar adelante sus propósitos subversivos por un tiempo en relativa paz (Bey, 1985, párr. 15).

\section{El cuerpo politizado}

Tanto la acción mencionada de Grupal Crew Collective como en su conjunto y en líneas generales los procedimientos empleados en Reclaim the Streets, comparten como común denominador la presencia del cuerpo y de lo sonoro como forma de resistencia. Así sucedía también en las acciones anteriormente mencionadas de Lacy. Todas ellas surgen con un carácter eventual -como afirma Bey- y todas ellas lo hacen con relación a la manifestación y presencia de los cuerpos y a su capacidad de irrumpir en las lógicas del sistema. Sobre este tema en particular reflexiona John Jordan (2001), cofundador de Reclaim the Streets, en el siguiente fragmento:

Para implicarse en la acción directa uno ha de sentir la suficiente pasión como para poner sus valores en práctica; consiste literalmente en dar cuerpo [embody] a tus sentimientos, actuar [perform] tu política. El cuerpo ha sido marginado en nuestra cultura tecnocrática, lo cual entraña un peligro: revela una sociedad sin ningún tipo de contacto consigo misma y con su entorno, una sociedad que prefiere utilizar como metáfora la máquina constituida por piezas duras sin conexión antes que el cuerpo interconectado, fluido, blando. La acción directa hace visible el carácter devastador de la maquinaria de la cultura industrial y devuelve el cuerpo al centro de la política y de la práctica cultural. Cuando uno sitúa su cuerpo directamente en los engranajes de la máquina, introduce un punto de resistencia en la circulación del poder, transforma su propio cuerpo obligando a la sociedad industrial a explicar y justificar sus acciones (p. 372).

En las diferentes acciones que estamos mencionando a lo largo del artículo, el sonido es el elemento que activa los cuerpos provocando con ello. Es decir que, mediante la 
creación sonora, se alude explícitamente a una escucha social, que procura una sensibilización hacia algún aspecto en concreto que va más allá del sonido sí mismo.

\section{La escucha escenografiada}

Se puede concluir de esta aproximación que lo que procuran estos ejemplos mencionados, como muchos otros que serían susceptibles de aparecer también, es una escucha escenografiada, un encuentro con determinadas realidades mediado por el la creación sonora. Las acciones recurren a la escucha para llamar la atención sobre aspectos tan distintos como la invisibilidad de un colectivo o la resistencia ante una prohibición. Con ello ponen en tela de juicio las fronteras entre lo público y lo privado, entre lo que existe y tiene una representación pública y lo que es ignorado, ocultado o silenciado.

Este acercamiento se hace más rico si lo completamos con el análisis de otras acciones artísticas donde este hecho se hace algo explícito. Es el caso del performance de Sharon Hayes, Everything Else Has Failed! Don't You Think It's Time For Love?, en la que abordó la cuestión de la privacidad y su relación con el espacio público. En el performance la artista amplificó su voz en las calles de Nueva York para hablar a un amante anónimo durante varios días en diferentes localizaciones del barrio financiero de esta ciudad. Al insertar amplificado un discurso privado en el ámbito de lo público, la acción evocaba preguntas acerca del territorio y los límites de expresión de lo público y lo privado, abriendo el discurso hacia las cuestiones de género, afecto y normalización, y a sus formas de representación en el espacio público.

Estas escenografías para la escucha conllevan en prácticamente todos los casos crear las condiciones para una escucha amplificada, ya sea a través de aparatos específicos o mediante las propias condiciones en que queda organizado el marco para la escucha. La escucha amplificada invita a considerar aquello que va más allá de lo que se oye, una escucha que permite, en otras palabras, atender a las cuestiones que evoca aquello que se escucha. Esta noción tan dilatada de amplificación introduce la cuestión de la escucha sociológica a la que se refiere Les Back en su ensayo The Art of Listening (2010): un gesto que permite escuchar en sentido figurado lo oculto y lo invisible. Una escucha que sirve como desencadenante para percibir la espacialidad de un contexto y por lo tanto también para conocerla. A este aspecto se refiere, también, Brandon LaBelle (2010) que añade además, derivado de ello, el aspecto revelador del sonido como vía para el conocimiento, a un mismo tiempo, personal y colectivo:

El conocimiento auditivo es un empuje epistemológico que despliega un evento espacio-temporal. El sonido abre un campo de interacción. Se convierte en un canal, un fluido, un flujo de voz, de juego, de drama, de lo mutuo y compartido para forjar una micro-geografía del momento mientras se desvanece como una propagación.

ESCENA. Revista de las artes, 2018, Vol. 78, Núm. 1 (julio-diciembre), pp. 70-82. ISSN 2215-4906 
Desde mi perspectiva, esto hace del sonido un modelo significativo para pensar y experimentar la condición contemporánea, como una espacialidad relacional tal y como demanda la cultura global y que necesita una continua reformulación. Nos ubica en un entorno extremadamente animado y energético que, como el fenómeno aural, habitualmente sobrepasa los parámetros convencionales y las posibilidades de representación ...

Las dinámicas del conocimiento auditivo ofrecen una oportunidad clave para moverse en el momento presente al crear espacios compartidos que no pertenecen a una única persona y que aún así producen una sensación de intimidad. El sonido siempre me pertenece y no me pertenece (pp. xvii - xviii).

Resulta tremendamente esclarecedora esta afirmación de LaBelle para apoyar la tesis con la que comenzaba este artículo: la idoneidad del sonido para crear las condiciones que posibiliten un conocimiento posterior personal y compartido. Unas condiciones que por las propias cualidades del sonido, que fluye y que se propaga, lleva a pensar las obras desde los procesos aprovechando la temporalidad como un recurso que permite amoldarse o reaccionar a los contextos específicos tal y como se ha observado en las acciones previamente mencionadas.

Ninguna de ellas estaba orientada al objeto, como tampoco ninguna de ellas presentaba una acción argumentalmente cerrada. No lo hacía Grupal Crew Collective en ZAA Soundsystem cuyo objeto -el sistema de sonido y el acto de pinchar música y bailar- mostrado en la excepcionalidad del espacio público irradiaba hacia la situación política concreta; tampoco era el objeto el fin último de las acciones de Suzanne Lacy cuando hablaba por la radio, o cuando reunió a las mujeres para conversar en la playa. Las acciones buscaban por el contrario provocar y crear reacciones, no tanto ofrecer argumentos a una cuestión en particular. Eso las hacía asimismo adquirir según los casos un carácter activista o podría decirse también de activación del espacio urbano que cobra sentido en el proceso mismo de realización y recepción, lo que conduce en en este punto a pensar la acción sonora como un trabajo de campo, con un carácter exploratorio y experimental, que permite recabar datos y conocer sobre el terreno.

En esta línea se inscribe la acción colectiva Immigrant Sounds (Sonidos inmigrantes) iniciada por Abelardo Gil-Fournier y mi persona en las calles de Estocolmo, en las que se invitó a explorar el entorno urbano portando un pequeño dispositivo provisto de un sonido semejante al de una cigarra. El dispositivo permitía al participante controlar el volumen del sonido (dentro de un rango previamente determinado) sin tener acceso a eliminarlo por completo siendo la intención que cada participante realizara la exploración sintiéndose señalado públicamente por su identidad y condición (sonoras en este caso) a través de la escucha de los otros. De este modo, se pretendía reflexionar sobre las convenciones y las realidades de los inmigrantes a través de los comportamientos de los participantes durante 
la exploración, dando lugar a determinadas reflexiones sobre las políticas migratorias y la acogida ciudadana de estas comunidades. En este caso era el sonido procedente de tierras más áridas y calurosas el que marcaba y señalaba a los participantes, un sonido bastante molesto en su continuidad que condicionaba la exploración y era rápidamente identificado por la escucha de los otros, ajenos a la acción. Ello ponía a los participantes en el punto de mira de los otros ciudadanos (con la incomodidad que ello suponía) haciéndoles reproducir inconscientemente ciertos patrones de comportamiento de las comunidades inmigrantes como el agrupamiento por semejanza, el intento de pasar desapercibido, la ocultación de los rasgos predominantes y otros detalles interesantes.

\section{Conclusión}

Lo que se pone de manifiesto a través de las acciones y piezas comentadas es que al centrar la atención no tanto en los objetos sonoros resultantes (en el conocimiento ya producido al que apuntaba Sterne) cuanto en los procesos que abre la creación sonora, se aprecian metodologías que inician procesos de reconocimiento o de reflexión, procesos que llaman la atención sobre aspectos determinados y que muchas veces tienen un eco en la cultura sonora popular y en la formas en que habitamos y escuchamos o fijamos la atención en el espacio público. Los procesos que abren persiguen en la mayoría de los casos un mismo cometido: la posibilidad de hacer, es decir la capacidad como sujetos de intervenir el espacio público y asistir a lo que ello implica. Se hace así evidente la potencialidad que tiene el gesto, la acción en el espacio público como brecha disruptiva. En este marco la intervención sonora, o en términos mucho más amplios pero igualmente válidos, la creación sonora, consigue provocar reacciones en el espacio público que conducen a su conocimiento. A esta posibilidad se vincula inevitablemente una imposibilidad de prever los resultados de dichas acciones que suceden en el tiempo real del transcurso cotidiano y por lo tanto también una imposibilidad de prever el conocimiento que puede surgir de ellas. La relevancia precisamente de estas prácticas sonoras en el espacio público es precisamente ese encuentro latente y realizable con los procesos y los sujetos ya presentes, desde los que adquiere todo su sentido y sin los que se podría considerar su relevancia artística.

\section{Referencias}

Back, L. (2007). The Art of Listening. Oxford: Bloomsbury.

Bey, H. (1985). Zona Temporalmente Autónoma. Recuperado de http://www.merzmail.net/zona.htm

Blanco, P. (2001). Explorando el terreno. En P. Blanco, J. Carrillo, J. Claramonte \& M. Expósito (Eds.), Modos de hacer. Arte crítico, esfera pública y acción directa (pp. 23-50). Salamanca: Ediciones Universidad de Salamanca.

ESCENA. Revista de las artes, 2018, Vol. 78, Núm. 1 (julio-diciembre), pp. 70-82. ISSN 2215-4906 
Delgado, M. (2008). Apropiaciones Inapropiadas. Usos insolentes del espacio público en Barcelona. Recuperado de http://www.ciutatsocasionals.net/textos/textosprincipalcast/ delgado.htm

Jordan, J. (2001). El arte de la necesidad: La imaginación subversiva del movimiento de oposición a las carreteras y Reclaim the Streets. En P. Blanco, J. Carrillo, J. Claramonte \& M. Expósito (Eds.), Modos de hacer. Arte crítico, esfera pública y acción directa (p. 372). Salamanca: Ediciones Universidad de Salamanca.

LaBelle, B. (2010). Acoustic Territories. Sound Culture and Everyday Life. Nueva York / Londres: Continuum

Lacy, S. (1994a). Cultural Pilgrimages and Metaphoric Journeys. En S. Lacy (Ed.), Mapping the Terrain. New Genre Public Art (pp. 19-30). Seattle / Wash: Bay Press.

Lacy, S. (1994b). Debated Territory: Toward a Critical Language for Public Art. En S. Lacy (Ed.), Mapping the Terrain. New Genre Public Art (pp. 171-185). Seattle / Wash: Bay Press.

Sterne, J. (2012). Sonic Imaginations. En The Sound Studies Reader (pp. 1-17). Nueva York: Routledge.

Voegelin, S. (2011). Listening to Noise and Silence. Towards a Philosophy of Sound Art. Nueva York / Londres: Continuum 